\title{
Disseminated BCG disease in an infant with severe combined immunodeficiency
}

\author{
Anirban Mandal ${ }^{*}$, Amitabh Singh ${ }^{2}$, Puneet Kaur Sahi ${ }^{3}$ and Bhavika Rishi ${ }^{4}$ \\ ${ }^{1}$ Department of Pediatrics, Sitaram Bhartia Institute of Science and Research, New Delhi, India \\ ${ }^{2}$ Department of Pediatrics, Chacha Nehru Bal Chikitsalaya, New Delhi, India \\ ${ }^{3}$ Department of Pediatrics, Kalawati Saran Children's Hospital, New Delhi, India \\ ${ }^{4}$ Department of Pathology, Lady Harding Medical College, New Delhi, India
}

\begin{abstract}
BCG (Bacillus Calmette Guerin) being a live attenuated vaccine may cause disseminated disease (BCGiosis) in patients with impaired immunity. Patients with severe combined immunodeficiency (SCID) having defect in both cellular and humoral immunity are predisposed to a host of live vaccine related complications, especially BCG.
\end{abstract}

We report an infant presenting persistent fever, weight loss, anemia, hepatosplenomegaly, skin rash and multiple osteolytic lesions subsequently diagnosed with SCID and BCGiosis.

The case highlights the possible risk of such rare yet lethal complication of BCG especially where it is given routinely at birth or in the neonatal period and also emphasizes the need for neonatal screening for SCID in such regions.

\section{Introduction}

Bacillus Calmette Guerin (BCG) vaccine, made from an attenuated strain of Mycobacterium bovis, is administered routinely in the neonatal period in TB endemic countries including India, as a part of the Universal Immunization schedule. Having only local complications in general, BCG is associated with lethal disseminated disease in those with immunodeficiency [Lott 1984, Talbot 1997]. Several primary immunodeficiency syndromes has been associated with disseminated BCG disease (BCGiosis), such as Mendelian susceptibility to Mycobacterial disease (MSMD), hyper-IgM syndrome, Di George syndrome, chronic granulomatous disease, Severe combined Immunodeficiency (SCID), IL (Interleukin)-12/23 receptor $\beta 1$ chain deficiency, IL-12p40 deficiency, STAT1 (Signal transducer and activator of transcription 1) deficiency and NEMO (Nuclear factor kappa-beta essential modulator) deficiency [2-4]. SCID is arguably the most severe and lethal inherited primary immunodeficiency with a prevalence of approximately 1 in 50,000 live births. It is aptly called as a pediatric emergency as it invariably leads to fatality in infancy without early aggressive therapy and Hematopoietic stem cell transplantation (HSCT) or other specific therapy [5].

We present an infant with SCID and BCGiosis. Our case describes the very rare yet possible risk of such a devastating complication of BCG, especially important for countries where it is given routinely; it also emphasis the need for screening of newborns for SCID in similar regions.

\section{Case report}

A-6 month-old baby boy was admitted with complaints of fever for 5 months, generalized rash involving palms and soles for 3 months, cough and cold for 1 month, poor feeding and weight loss over last
1 month. He received multiple courses of oral antibiotics with no response. There was no history of vesico-bullous lesion, mucosal involvement, itching or similar lesions in any other family members. There was also no history of contact with $\mathrm{TB}$, ear discharge, seborrhea or blood component therapy. He was product of non-consanguineous marriage, born to a primigravida at term gestation by normal vaginal delivery with a birth weight of $3.35 \mathrm{~kg}$. There was no history of chronic cough or infertility in the mother before conception and there was no antenatal history of fever with/ without rash or genital ulcers. There were no perinatal concerns and he remained apparently asymptomatic and well thriving on exclusive breastfeeding till 2 months of age. $\mathrm{He}$ received BCG and OPV (oral polio vaccine) at birth and was vaccinated till 10 weeks of age as per the National immunization schedule. On examination, he had stable vital parameters with mild pallor but no icterus or lymphadenopathy. Anthropometric parameters were suggestive of moderate wasting. There were erythematous papules with central crusting and scanty sero-sanguinous discharge (Figure 1a and 1b). The BCG site was still active with a crusted lesion and scanty serous discharge (Figure1c). Systemic examination was remarkable with spleno-hepatomegaly but other systems were essentially normal. Initial investigations were suggestive of microcytic, hypochromic anemia,

Correspondence to: Anirban Mandal, Department of Pediatrics, Sitaram Bhartia Institute of Science and Research, New Delhi, India Telephone: 8826836670; Email: anirban.nrs@gmail.com

Key words: primary immunodeficiency, adverse event following immunization, hematopoietic stem cell transplantation, neonatal screening

Received: August 27, 2016; Accepted: September 22, 2016; Published: September 27, 2016 
while rest was non-contributory (Table 1). So, skin biopsy was done from the lesions and it revealed irregular acanthosis and papillomatosis; dermis showing dense acute and chronic inflammatory infiltrate with collection of foamy histiocytes spilling into the subcutaneous tissue and modified Ziehl Nelson stain for AFB was positive (Figure 2a). MGIT (Mycoacterium growth indicator tube) culture of skin biopsy grew Mycobacterium tuberculosis complex. Skeletal survey showed multiple lytic lesion involving both long and short bones without sclerosis (Figure 3). Contrast enhanced computed tomography (CECT) of abdomen showed enlarged liver and spleen with multiple hypodence lesions and peripancreatic adenopathy (Figure $2 b$ ); while, CT brain and chest were normal. He was started on category 1 four drug antitubercular therapy along with nutritional rehabilitation and other supportive measures. But his fever persisted with worsening of general condition. Further immunological investigations carried out were suggestive of Severe Combined Immunodeficiency (SCID) (Table 2). Mutational analysis could not be done due to unavailability. He was given intravenous immunoglobulin (IVIG) and was also started on antifungal (Fluconazole) and antibacterial (Cotrimoxazole) prophylaxis. In the

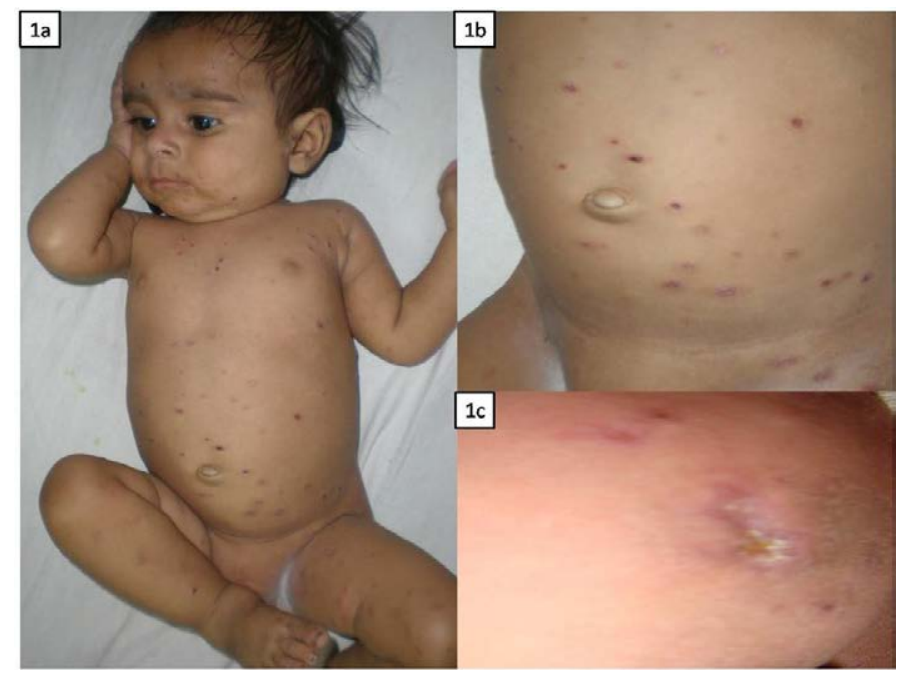

Figure 1a. 6-month-old-boy with mild pallor, moderate wasting and generalized rash; 1b - Papulo-nodular, erythematous rash with central crusting over abdomen; 1c-Active BCG scar over left deltoid region showing crusting and scanty serous discharge. mean time species typing of the isolated mycobacterium revealed Mycobacterium bovis confirming the diagnosis of disseminated BCG disease. The antitubercular therapy was modified to replace Pyrizinamide with Levofloxacin and addition of Amikacin. Gradually the child improved and bone marrow transplantation was planned. On follow up till 2 months after discharge, he remained afebrile, gained weight and most of the skin lesions also healed; but he was lost to follow up after that.

\section{Discussion}

Our patient had persistent fever, weight loss, anemia, hepatosplenomegaly, skin rash and multiple osteolytic lesions. Initial possibilities considered were congenital syphilis, congenital TB/ acquired disseminated TB, Langerhan's cell histiocytosis (LCH), metastatic neuroblastoma and hematological malignancies. Non invasive investigations being non-contributory, skin biopsy and subsequent culture results confirmed the diagnosis of mycobacterial infection. Prolonged history, atypical clinical features and radiological involvement coupled with poor response to appropriate therapy prompted us to look for an underlying immunodeficiency and established the diagnosis of SCID even before the final diagnosis of BCGiosis was made.

Though there is no established definition, our patient fulfilled the criteria for a 'definitive' case of disseminated BCG infection as per the

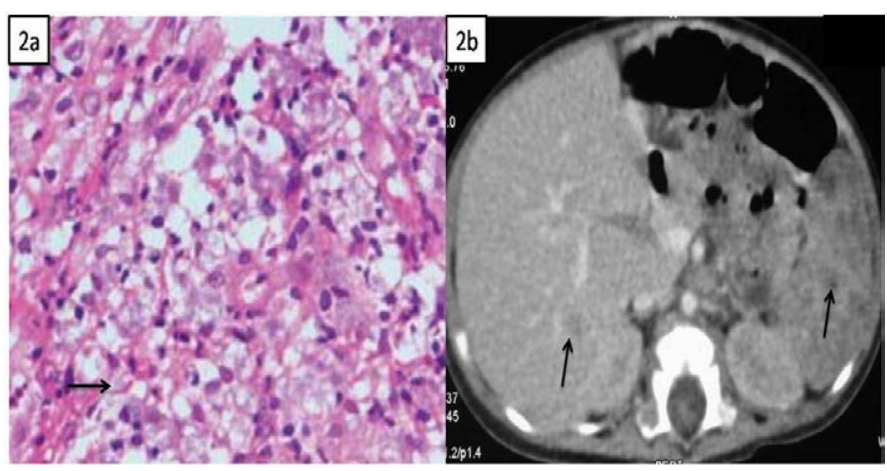

Figure 2a. Modified Ziehl Nelson staining of skin biopsy showing AFB (black arrow); $\mathbf{2 b}-$ CECT of abdomen showing enlarged liver and spleen with hypodense lesions (black arrow).

Table 1. Initial investigations of the child.

\begin{tabular}{|c|c|c|c|}
\hline Investigation & Result & Investigation & Result \\
\hline Hemoglobin (gm/dl) & 7.9 & $\begin{array}{l}\text { Serum glutamic-oxaloacetic transaminase } \\
(\text { SGOT })(\mathrm{U} / \mathrm{L})\end{array}$ & 36 \\
\hline Total leukocyte count $\left(/ \mathrm{mm}^{3}\right)$ & 9600 & $\begin{array}{l}\text { Serum glutamic pyruvic transaminase } \\
\text { (SGPT) (U/L) }\end{array}$ & 33 \\
\hline Differential leukocyte count & $\begin{array}{c}\text { Neutrophil } 76 \% \text {, Lymphocyte } 19 \% \text {, Monocyte } \\
4 \% \text {, Eosinophil } 1 \%\end{array}$ & Alkaline phosphatase (U/L) & 312 \\
\hline Platelet count & $2.9 \mathrm{lakh} / \mathrm{mm}^{3}$ & Calcium (mg/dl) & 8.9 \\
\hline Peripheral smear & $\begin{array}{l}\text { Microcytic, hypochromic red blood cells with } \\
\text { marked aniso-poikilocytosis; no abnormal } \\
\text { cells/ blasts }\end{array}$ & Phosphate (mg/dl) & 4.6 \\
\hline Sodium $(\mathrm{mEq} / \mathrm{L})$ & 141 & Blood culture & Sterile \\
\hline Potassium (mEq/L) & 5.0 & Mantoux test & $0 \mathrm{~mm}$ \\
\hline Urea (mg/dl) & 28 & $\begin{array}{l}\text { VDRL (Venereal Disease Research } \\
\text { Laboratory) }\end{array}$ & Negative \\
\hline Creatinine (mg/dl) & 0.3 & $\begin{array}{c}\text { TPHA (Treponema pallidum } \\
\text { haemagglutination) test of mother }\end{array}$ & Negative \\
\hline Total bilirubin/ conjugated ( $\mathrm{gm} / \mathrm{dl})$ & $0.5 / 0.1$ & HIV ELISA of mother & Negative \\
\hline Total protein $(\mathrm{gm} / \mathrm{dl})$ & 5.9 & Chest x-ray & Normal \\
\hline Albumin $(\mathrm{gm} / \mathrm{dl})$ & 3.5 & Gastric aspirate for acid fast bacilli & Negative \\
\hline
\end{tabular}




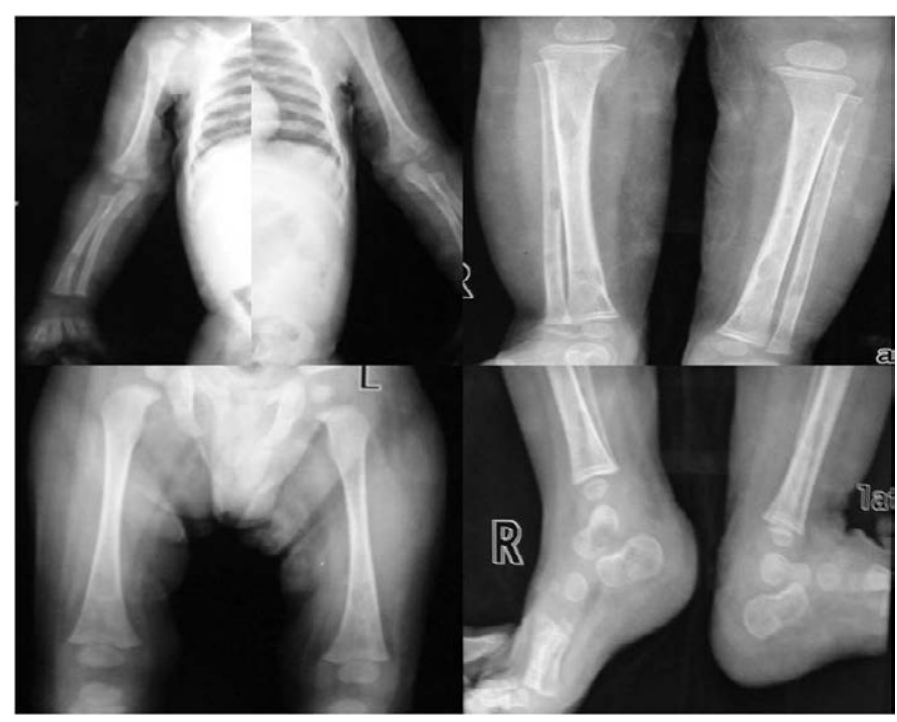

Figure 3. skeletal survey showing osteolytic lesions with periosteal reaction in both long and short bones.

Table 2. Immunological investigations of the child.

\begin{tabular}{|c|c|c|}
\hline Investigation & Result & Reference \\
\hline Immunoglobulin $\mathrm{G}(\mathrm{mg} / \mathrm{dl})$ & 112 & $217-964$ \\
\hline Immunoglobulin $\mathrm{A}(\mathrm{mg} / \mathrm{dl})$ & 7 & $34-126$ \\
\hline Immunoglobulin $\mathrm{M}(\mathrm{mg} / \mathrm{dl})$ & 3 & $11-90$ \\
\hline Absolute lymphocyte count $\left(/ \mathrm{mm}^{3}\right)$ & 1188 & $5300-6700$ \\
\hline CD3+ T lymphocyte $\left(/ \mathrm{mm}^{3}\right)$ & 1 & $3400-4600$ \\
\hline $\mathrm{CD} 4+\mathrm{T}$ lymphocytes $\left(/ \mathrm{mm}^{3}\right)$ & 0 & $2600-3500$ \\
\hline $\mathrm{CD} 8+\mathrm{T}$ lymphocytes $\left(/ \mathrm{mm}^{3}\right)$ & 1 & $390-2500$ \\
\hline $\mathrm{CD} 19+\mathrm{T}$ lymphocytes $\left(/ \mathrm{mm}^{3}\right)$ & 17 & $11-45$ \\
\hline $\mathrm{CD} 16 / 56+\mathrm{T}$ lymphocytes $\left(/ \mathrm{mm}^{3}\right)$ & 2 & $11-24$ \\
\hline
\end{tabular}

proposed diagnostic criteria [2,6]. Our child had most of the frequently described manifestations of BCGiosis, i.e., fever, weight loss, anemia, skin rash and organomegaly but lymphadenopathy was conspicuously absent [2,7]. Skin manifestations have been an interesting mode of presentation in BCGiosis with a wide range of lesions being described ranging from macula-papules, subcutaneous nodules and ulcers $[2,7,6]$. Our case had lesions reminiscent of LCH and skin biopsy came to the aid. Indeed, skin biopsy has been shown to be a critical investigation in diagnosis of BCGiosis and may also have role in prognostication as well [8].

In a review of adverse events following immunization (AEFI) in cases of primary immunodeficiency [9] patients with SCID and CGD had the greatest percentage of serious AEFI with BCG being the most commonly incriminated vaccine. Complications of BCG including BCGiosis have been described with all the genetic forms of SCID with no identifiable difference between various types [4]. In a recent review [10] of 349 BCG-vaccinated patients with SCID from 17 countries $51 \%$ of patients had BCG-associated complications, $34 \%$ disseminated and $17 \%$ localized. BCG associated complications and death were more common in children receiving early $(<1$ month) vaccination. Interestingly, BCG-associated complications were reported in only 2 of
78 patients who received antitubercular therapy before symptom onset with no deaths caused by BCG-associated complications. In contrast, 46 BCG-associated deaths were reported among 160 patients treated with antitubercular therapy for a symptomatic BCG infection. Even after HSCT, immune reconstitution syndrome (IRS) remains an important cause of morbidity and mortality in this group of SCID patients.

Data from Brazil, a country with high burden of TB, where routine BCG immunization is administered in neonatal period reveals that around $86 \%$ of children received BCG before their SCID diagnosis and $41 \%$ developed BCGiosis; half of the patients died with BCGiosis being the predominant cause [11].

\section{Conclusion}

In countries with routine BCG administration in infancy BCGiosis is an important mode of presentation of primary immunodeficiencies, especially SCID. To avoid delayed diagnosis of SCID and the organ damage secondary to BCGiosis, neonatal screening to identify lymphopenia should be considered. On the other hand patients, who already received BCG before diagnosis of SCID, may be started on antitubercular therapy along with standard management guidelines $[5,10]$ even before symptom onset.

\section{References}

1. Lotte A, Wasz-Höckert O, Poisson N, Dumitrescu N, Verron M, et al. (1984) BCG complications. Estimates of the risks among vaccinated subjects and statistical analysis of their main characteristics. Adv Tuberc Res 21: 107-193. [Crossref]

2. Talbot EA, Perkins MD, Silva SF, Frothingham R (1997) Disseminated bacilleCalmetteGuérin disease after vaccination: case report and review. Clin Infect Dis 24: 1139-1146. [Crossref]

3. Sadeghi-Shanbestari M, Ansarin K, Maljaei SH, Rafeey M, Pezeshki Z, et al. (2009) Immunologic aspects of patients with disseminated bacilleCalmette-Guerin disease in north-west of Iran. Ital J Pediatr 35: 42. [Crossref]

4. Norouzi S, Aghamohammadi A, Mamishi S, Rosenzweig SD, Rezaei N, et al (2012) Bacillus Calmette-Guérin (BCG) complications associated with primary immunodeficiency diseases. $J$ Infect 64: 543-554. [Crossref]

5. Madkaikar M, Aluri J, Gupta S3 (2016) Guidelines for Screening, Early Diagnosis and Management of Severe Combined Immunodeficiency (SCID) in India. Indian J Pediatr 83: 455-462. [Crossref]

6. Bernatowska EA, Wolska-Kusnierz B, Pac M, Kurenko-Deptuch M, Zwolska Z, et al (2007) Disseminated bacillus Calmette-Guérin infection and immunodeficiency. Emerg Infect Dis 13: 799-801. [Crossref]

7. Shahmohammadi S, Saffar MJ, Rezai MS (2014) BCG-osis after BCG vaccination in immunocompromised children: Case series and review. J Pediatr Rev 2: 47-54.

8. Gantzer A, Neven B, Picard C, BrousseN, Lortholary O, et al. (2014) Severe cutaneous bacillus Calmette-Guérin infection in immunocompromised children: the relevance of skin biopsy. J Cutan Pathol 40: 30-37. [Crossref]

9. Sarmiento JD, Villada F, Orrego JC, Franco JL,Trujillo-Vargas CM, et al. (2016) Adverse events following immunization in patients with primary immunodeficiencies. Vaccine 34: 1611-1616. [Crossref]

10. Marciano BE, Huang CY, Joshi G, Rezaei N, Carvalho BC, et al. (2014) BCG vaccination in patients with severe combined immunodeficiency: complications, risks, and vaccination policies. J Allergy Clin Immunol 133:1134-1141. [Crossref]

11. Mazzucchelli, JT, Bonfim, C, Castro, GG, Condino-Neto, AA, Costa NM, et al. (2014) Severe combined immunodeficiency in Brazil: management, prognosis, and BCGassociated complication. JInvestig Allergol Clin Immunol 24: 184-191.

Copyright: (C2016 Mandal A. This is an open-access article distributed under the terms of the Creative Commons Attribution License, which permits unrestricted use, distribution, and reproduction in any medium, provided the original author and source are credited. 